\title{
Neutron-induced nuclear data for the MYRRHA fast spectrum facility
}

\author{
Pablo Romojaro ${ }^{1, a}$, Gašper Žerovnik ${ }^{2}$, Francisco Álvarez-Velarde ${ }^{3}$, Alexey Stankovskiy ${ }^{4}$, Ivan Kodeli ${ }^{5}$ Luca Fiorito $^{4}$, \\ Carlos Javier Díez ${ }^{6}$, Óscar Cabellos ${ }^{6}$, Nuria García-Herranz ${ }^{1}$, Jan Heyse ${ }^{2}$, Carlos Paradela ${ }^{2}$, Peter Schillebeeckx ${ }^{2}$, \\ and Gert Van den Eynde ${ }^{4}$ \\ 1 UPM, Energy Engineering Department, C/ José Gutiérrez Abascal 2, 28006 Madrid, Spain \\ 2 EC-JRC Geel, Standards for Nuclear Safety, Security and Safeguards, Retieseweg 111, 2440 Geel, Belgium \\ 3 CIEMAT, Nuclear Innovation Unit, Avda. Complutense 40, 28040 Madrid, Spain \\ 4 SCK•CEN, Institute for Advanced Nuclear Systems, Boeretang 200, 2400 Mol, Belgium \\ 5 JSI, Reactor Physics Department, Jamova cesta 39, 1000 Ljubljana, Slovenia \\ ${ }^{6}$ OECD NEA, Data Bank, Quai Alphonse Le Gallo 46, 92100 Boulogne-Billancourt, France
}

\begin{abstract}
The MYRRHA (Multi-purpose hYbrid Research Reactor for High-tech Applications) concept is a flexible experimental lead-bismuth cooled and mixed-oxide (MOX) fueled fast spectrum facility designed to operate both in sub-critical (accelerator driven) and critical modes. One of the key issues for the safe operation of the reactor is the uncertainty assessment during the design works. The main objective of the European project CHANDA (solving CHAllenges in Nuclear DAta) Work Package 10 is to improve MYRRHA relevant nuclear data in order to reduce the reactor parameter uncertainties derived from them. In order to achieve this goal, several tasks have been undertaken. First, a sensitivity study of MYRRHA integral parameters, such as energy dependent cross sections, fission spectra and neutron multiplicities, to nuclear data has been conducted resulting in a list of MYRRHA relevant quantities (nuclides and reactions). On the second task, an analysis of the existing experimental data and evaluations for the quantities included in the list has been carried out. In this framework, the impact on the multiplication factor of quantities from different nuclear data libraries for different nuclides, reactions and energy regions has been investigated on the MYRRHA MOX critical core model. As the next step, new experiments and evaluations will be performed in order to improve existing nuclear data libraries.
\end{abstract}

\section{Introduction}

MYRRHA is a flexible experimental facility being designed at $\mathrm{SCK} \cdot \mathrm{CEN}$, Mol, Belgium [1]. It is conceived to operate both in sub-critical, or Accelerator Driven System (ADS) mode, driven by a $600 \mathrm{MeV}$ linear proton accelerator, and in critical mode, as a leadbismuth cooled fast reactor. An accurate knowledge of the safety parameters (i.e., neutron multiplication factor $k_{\text {eff }}$, reactivity coefficients, etc.) is required considering the complex neutronic design of MYRRHA [2]. In the neutronic and safety design of the reactor, an important aspect is the uncertainty propagation and sensitivity to nuclear data; therefore, accurate and reliable nuclear data are needed for MYRRHA.

The EC FP7 CHANDA project [3], whose acronym stands for "solving CHAllenges in Nuclear DAta", addresses the challenges in the field of nuclear data for nuclear applications. This project will allow European scientists and institutions to improve the nuclear data needed for simulation tools in order to increase the accuracy of code assessments and consequently to better focus the design of expensive experiments. In particular,

a e-mail: pablo.romojaro@externos.ciemat.es the main objective of Work Package 10 is to improve MYRRHA-relevant nuclear data in order to reduce the reactor parameter uncertainties derived from them.

Within this framework, a neutron-induced nuclear data sensitivity and uncertainty $(\mathrm{S} / \mathrm{U})$ analysis of the latest MYRRHA design has been performed as a first task and a list of the most important nuclides and quantities has been obtained. As a second task, a detailed analysis of the existing experimental data and evaluations for the quantities included in the list has been carried out, investigating the impact on $k_{\text {eff }}$ of quantities from different existing nuclear data libraries for different nuclides, reactions and energy regions on the MYRRHA MOX critical core model. Bismuth and lead have been chosen as the main objects of study for improvement of nuclear data for MYRRHA since they are of vital importance and are not covered by the CIELO pilot project [4]. New experimental data in the resonance region will be obtained at the GELINA facility.

In this paper, the results of the $\mathrm{S} / \mathrm{U}$ analysis for the MOX critical core are presented. An overview of the experimental data and existing evaluations for $\mathrm{Pb}$ and $\mathrm{Bi}$ is given and some issues spotted in the evaluations are discussed. 


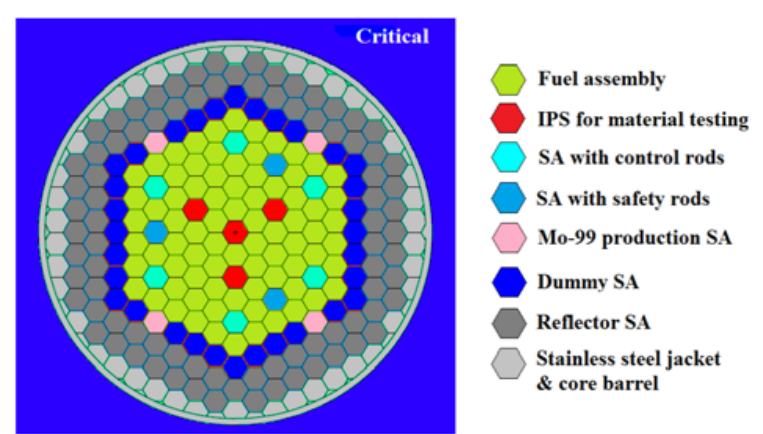

Figure 1. Critical MYRRHA core layout.

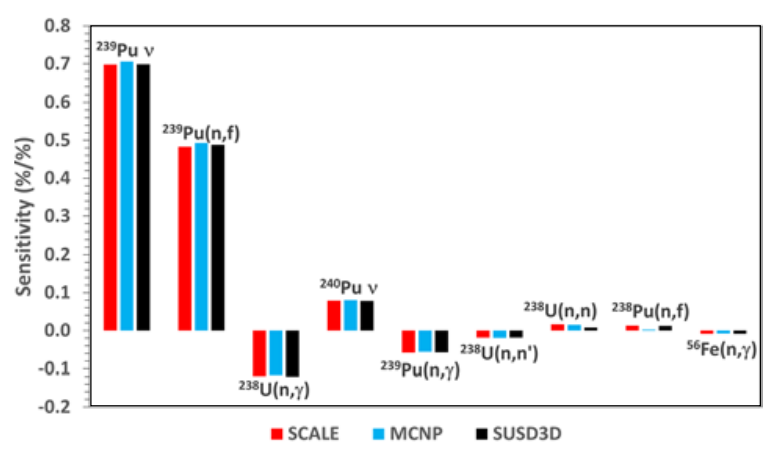

Figure 2. ISC for MYRRHA obtained by the different simulation codes.

\section{Sensitivity and uncertainty analysis}

MYRRHA's updated core design is described in detail in Ref. [5]. For this study a simplified model [6], homogenised on fuel assembly level, has been used. The layout of the core is shown in Fig. 1.

The procedures included in some of the state-of-theart computer codes dedicated to reactor core analyses have been employed in this work. In particular, the calculations have been performed with the SCALE6.2 system [7], in its third beta release, the MCNP6.1 code [8] and the SUSD3D code [9], part of the XSUN-2013 system [10]. Evaluated neutron-induced data from ENDF/B-VII.0 [11] have been used by SCALE and MCNP, and from ENDF/B-VII.1 [12] by SUSD3D. Additionally, a cylindrical geometry model of MYRRHA has been constructed to be used in the transport calculations performed by SUSD3D. This model has been developed using equivalent concentric cylinders conserving the total mass of each material, so no further homogenization was required, and adjusting the arrangement of the cylinders in order to represent the MYRRHA critical core configuration with high-fidelity.

The sensitivity analysis allows identifying the most important nuclides and reactions from the criticality safety neutronic design point of view. In Fig. 2, the Integrated Sensitivity Coefficients (ISC) of the major contributors to $k_{\text {eff }}$ uncertainty due to uncertainties in nuclear data are represented.

The key quantities in MYRRHA neutronics are ${ }^{239} \mathrm{Pu} v,{ }^{239} \mathrm{Pu}(\mathrm{n}, \mathrm{f})$ and ${ }^{238} \mathrm{U}(\mathrm{n}, \gamma)$. The ISC values are very close between codes and libraries, most of them with a relative difference smaller than $4 \%$. However, ${ }^{238} \mathrm{U}(\mathrm{n}, \mathrm{el})$, obtained by SUSD3D, has a difference of $51 \%$ to the value provided by SCALE, which can be attributed to omitting the implicit sensitivity contribution by SUSD3D. $25-83 \%$ differences (depending on MCNP methods) were observed
Table 1. $k_{e f f}$ uncertainty and main contributors for MYRRHA.

\begin{tabular}{cccc}
\hline \multirow{2}{*}{ Quantity } & \multicolumn{3}{c}{$\Delta \boldsymbol{k}_{\boldsymbol{e f f}} \boldsymbol{k}_{\boldsymbol{e} f \boldsymbol{f}}(\boldsymbol{\%})$} \\
\cline { 2 - 4 } & SCALE-6.0m & COMMARA-2 & JENDL-4.0 m \\
\hline${ }^{239} \mathrm{Pu} v$ & 0.701 & 0.056 & 0.112 \\
\hline${ }^{238} \mathrm{U}(\mathrm{n}, \mathrm{inl})$ & 0.324 & 0.331 & 0.148 \\
\hline${ }^{239} \mathrm{Pu}(\mathrm{n}, \gamma)$ & 0.265 & 0.273 & 0.194 \\
\hline${ }^{240} \mathrm{Pu} v$ & 0.227 & - & 0.016 \\
\hline${ }^{239} \mathrm{Pu}(\mathrm{n}, \mathrm{f})$ & 0.188 & 0.185 & 0.266 \\
\hline${ }^{239} \mathrm{Pu} \chi$ & 0.183 & - & 0.206 \\
\hline${ }^{238} \mathrm{Pu}(\mathrm{n}, \mathrm{f})$ & 0.160 & - & - \\
\hline${ }^{238} \mathrm{U}(\mathrm{n}, \gamma)$ & 0.152 & 0.157 & 0.196 \\
\hline${ }^{238} \mathrm{U}(\mathrm{n}, \mathrm{el})$ & 0.085 & 0.087 & 0.024 \\
\hline${ }^{238} \mathrm{U} v$ & 0.083 & 0.085 & 0.044 \\
\hline${ }^{56} \mathrm{Fe}(\mathrm{n}, \gamma)$ & 0.073 & - & 0.085 \\
\hline${ }^{56} \mathrm{Fe}(\mathrm{n}, \mathrm{el})$ & 0.049 & - & 0.137 \\
\hline${ }^{56} \mathrm{Fe}(\mathrm{n}, \mathrm{inl})$ & 0.032 & - & 0.098 \\
\hline Total XS & 0.922 & 0.516 & 0.512 \\
\hline Total PFNS ${ }^{(\mathbf{1})}$ & 0.210 & - & 0.210 \\
\hline TOTAL & $\mathbf{0 . 9 4 5}$ & $\sim \mathbf{0 . 5}$ & $\mathbf{0 . 5 5 3}$ \\
\hline
\end{tabular}

1) Prompt Fission Neutron Spectra.

between MCNP and SCALE for ${ }^{238} \mathrm{Pu}(\mathrm{n}, \mathrm{f})$. Furthermore for ${ }^{56} \mathrm{Fe}(\mathrm{n}, \gamma)$, differences of $20 \%$ were found between SUSD3D and SCALE, mostly due to different models used in SUSD3D (2D cylindrical) and SCALE (3D), and of $11 \%$ between MCNP and SCALE.

Excellent agreement was also observed in the calculated uncertainties, e.g. using the SCALE-6.0 [13] cross section covariance data the total uncertainties of 945 and $956 \mathrm{pcm}$ were calculated by the SUSD3D and SCALE codes, respectively. On the other hand, relatively large differences were found between different covariance matrices such as the SCALE-6.0m, JENDL$4.0 \mathrm{~m}$ [14] and COMMARA-2 [15] libraries. The principal contributors and the total uncertainty estimations based on the above covariance data are listed in Table 1 . The total uncertainty in $k_{\text {eff }}$ ranges between $\sim 0.5 \%$ (COMMARA-2 and JENDL-4.0 m) and $\sim 1 \%$ (SCALE$6.0 \mathrm{~m})$. SCALE- $6.0 \mathrm{~m}$ has in particular more conservative uncertainties in $v$ for ${ }^{239} \mathrm{Pu}$ and ${ }^{240} \mathrm{Pu}$.

Moreover, the $k_{\text {eff }}$ uncertainty resulting from a series of MCNP calculations with random data files has been evaluated. Since ${ }^{239} \mathrm{Pu}$ and ${ }^{238} \mathrm{U}$, due to their high content in MYRRHA MOX fuel, are major contributors to the neutron balance of the system, and have largest ISC of $k_{\text {eff }}$ to nuclear data (Fig. 2), the study was limited to the nuclear data uncertainty propagation of these two nuclides. A $k_{\text {eff }}$ uncertainty of $413 \mathrm{pcm}$ has been obtained due to covariance information for ${ }^{239} \mathrm{Pu}$ and ${ }^{238} \mathrm{U}$. This uncertainty is nearly half the total uncertainty of $\sim 1 \%$ calculated using the SCALE-6.0 covariances. However, it agrees quite well with the uncertainty due to ${ }^{239} \mathrm{Pu}$ and ${ }^{238} \mathrm{U}$ predicted by COMMARA-2 and JENDL$4.0 \mathrm{~m}$ (Table 1), $516 \mathrm{pcm}$ and $476 \mathrm{pcm}$ respectively, which confirms the conservativism of SCALE- 6.0 covariances.

\section{Comparison of evaluated libraries for lead and bismuth}

Taking into account the flexibility of the MYRRHA facility in providing various irradiation environments, in addition to the list of MYRRHA-relevant isotopes and reactions 


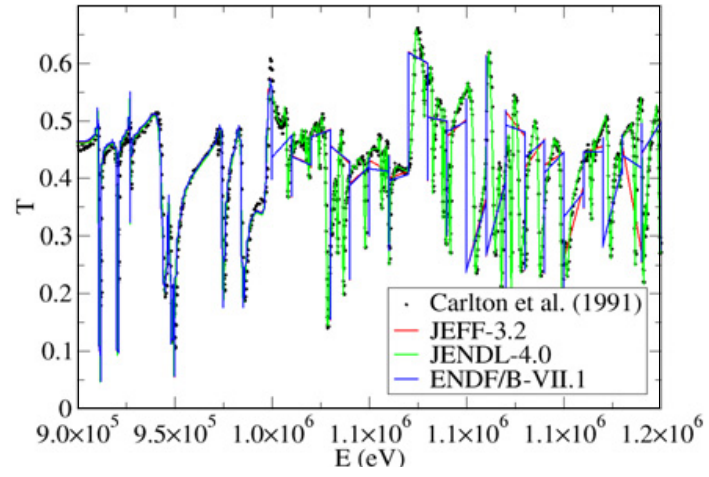

Figure 3. ${ }^{208} \mathrm{~Pb}$ comparison against experimental data.

presented in the previous section it is expedient to also consider the following quantities: ${ }^{208} \mathrm{~Pb}(\mathrm{n}, \mathrm{el})$ and (n,inl); and ${ }^{209} \mathrm{Bi}(\mathrm{n}, \gamma)$ and (n,inl).

To perform the comparison, the JEFF-3.2 [16] library has been taken as the reference. The other considered libraries are ENDF/B-VII.1 [11] and JENDL-4.0 [17]. The EXFOR database [18] has been used to search for experimental transmission time-of-flight (TOF) data for bismuth and lead, particularly focusing on experiments in the resolved and unresolved resonance energy ranges. The results available in EXFOR were derived from experimental data using the SAMMY code [19], so resolution and broadening were considered. The total cross section of each isotope contained in the nuclear data libraries has been converted to transmission data to reproduce the experiment using the following equation:

$$
T=e^{-n \sigma_{t o t}}
$$

where $\mathrm{T}$ is the transmission coefficient, $\mathrm{n}$ is the areal density of the sample and $\sigma_{\text {tot }}$ is the total cross section.

The impact of these libraries in the MYRRHA MOX critical core model has been evaluated by substituting the whole or individual reactions of JEFF-3.2, by the ones in ENDF/B-VII.1 or JENDL-4.0. The cross sections have been pre-processed using the NJOY processing system [20], while for the substitution of the data for individual reactions from different libraries the SANDY code [21] has been applied.

\section{1. ${ }^{208} \mathrm{~Pb}$}

Two transmission measurements were carried out by Carlton et al. [22] and Harvey et al. [23] at the ORELA facility with a $200 \mathrm{~m}$ flight path, using a ${ }^{208} \mathrm{~Pb}$ enriched sample with an areal density of 0.18594 at/b and ${ }^{6} \mathrm{Li}$ glass detectors and ${ }^{208} \mathrm{~Pb}$ enriched sample with an areal density of $0.16882 \mathrm{at} / \mathrm{b}$ and $\mathrm{Ne}-110$ plastic scintillators detectors, respectively. No information about the enrichment of the samples is provided in EXFOR. The ORELA facility is well characterized, including the resolution function. Carlton's experimental dataset has better experimental resolution in the MYRRHA-relevant energy range.

In Fig. 3, Carlton's transmission dataset is compared with the nuclear data evaluations. Perfect agreement with experimental data is found until the end of the resolved resonance region (RRR) $-1 \mathrm{MeV}$ - for all evaluations. In the unresolved resonance region (URR), JENDL-4.0 perfectly matches Carlton's data while the
Table 2. MCNP criticality calculations for the critical MOX configuration: multiplication factors for different nuclear data libraries for ${ }^{208} \mathrm{~Pb}$ reactions.

\begin{tabular}{lcccc} 
& \multicolumn{2}{c}{ ENDF/B-VII.1 } & \multicolumn{2}{c}{ JENDL-4.0 } \\
\hline $\begin{array}{l}\text { Reaction } \\
\text { substituted }\end{array}$ & $\boldsymbol{k}_{\text {eff }}$ & $\Delta \boldsymbol{k}_{\text {eff }}$ & $\boldsymbol{k}_{\text {eff }}$ & $\Delta \boldsymbol{k}_{\text {eff }}$ \\
\hline None & 1.00980 & 0 & 1.00980 & 0 \\
\hline all & 1.01014 & 0.00034 & 1.01083 & 0.00103 \\
\hline$(\mathrm{n}, \mathrm{n})$ & 1.00993 & 0.00013 & 1.00991 & 0.00011 \\
\hline$(\mathrm{n}, \mathrm{n} 1)$ & 1.00978 & -0.00002 & 1.00970 & -0.00010 \\
\hline$(\mathrm{n}, \gamma)$ & 1.00981 & 0.00001 & 1.00975 & -0.00005 \\
\hline
\end{tabular}

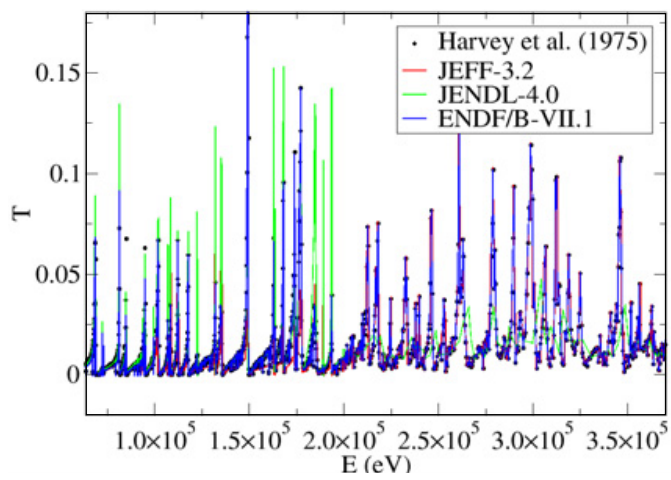

Figure 4. ${ }^{209} \mathrm{Bi}$ comparison against experimental data.

other evaluations also follow the experimental data but are interpolated within a coarser energy grid.

The results of differences in MOX critical core $k_{e f f}$ for ${ }^{208} \mathrm{~Pb}$ neutron induced reaction cross sections from different libraries are shown in Table 2. The statistical uncertainty in $k_{e f f}$ is less than $10 \mathrm{pcm}$ for all individual calculations and has been intentionally left out of the table. The differences using different libraries are (at least for some reactions) statistically significant, however of minor practical importance.

\section{2. ${ }^{209} \mathrm{Bi}$}

Harvey et al. [24] transmission data is the best documented data set available in the energy range of interest. The TOF experiment was performed at the ORELA facility in a $200 \mathrm{~m}$ flight path using a Bi sample with an areal density of $0.5651 \mathrm{at} / \mathrm{b}$ and Ne-110 plastic scintillator detectors. A comparison of the evaluated nuclear data libraries against experimental data is shown in Fig. 4.

JEFF-3.2, ENDF/B-VII.1 and JENDL-4.0 libraries have a good agreement with experimental data in the RRR $(10 \mu \mathrm{eV}-200 \mathrm{keV})$. In the URR and fast energy range, JEFF-3.2 and ENDF/B-VII.1 are coincidental with Harvey's data points while the JENDL-4.0 total cross section is fitted.

The results of differences in MOX critical core $k_{\text {eff }}$ for ${ }^{209} \mathrm{Bi}$ neutron induced reaction cross sections from different libraries are shown in Table 3. The higher multiplication factor for JEFF-3.2 can be attributed to the lack of the (negative) background cross section (in the elastic and total cross sections below $200 \mathrm{keV}$ ) which implies more efficient neutron slowing-down. For ENDF/B-VII.1, this difference is also partly due to differences in the capture cross section. Since the differences in the capture cross section occur in different energy regions and in different directions, a detailed 
Table 3. MCNP criticality calculations for the critical MOX configuration: multiplication factors for different nuclear data libraries for ${ }^{209} \mathrm{Bi}$ reactions.

\begin{tabular}{lcccc} 
& \multicolumn{2}{c}{ ENDF/B-VII.1 } & \multicolumn{2}{c}{ JENDL-4.0 } \\
\hline $\begin{array}{l}\text { Reaction } \\
\text { substituted }\end{array}$ & $\boldsymbol{k}_{\text {eff }}$ & $\Delta \boldsymbol{k}_{\text {eff }}$ & $\boldsymbol{k}_{\text {eff }}$ & $\Delta \boldsymbol{k}_{\text {eff }}$ \\
\hline None & 1.00980 & 0 & 1.00980 & 0 \\
\hline all & 1.00892 & -0.00088 & 1.00800 & -0.00180 \\
\hline$(\mathrm{n}, \mathrm{el})$ & 1.00864 & -0.00116 & 1.00842 & -0.00138 \\
\hline$(\mathrm{n}, \mathrm{n} 1)$ & 1.01012 & 0.00032 & 1.00973 & -0.00007 \\
\hline$(\mathrm{n}, \mathrm{n} 2)$ & 1.00956 & -0.00024 & 1.00991 & 0.00011 \\
\hline$(\mathrm{n}, \gamma)$ & 1.00838 & -0.00142 & 1.01019 & 0.00039 \\
\hline$(\mathrm{n}, \gamma)<200 \mathrm{keV}$ & 1.00836 & -0.00144 & 1.00948 & -0.00032 \\
\hline$(\mathrm{n}, \gamma) 100-200 \mathrm{keV}$ & 1.00902 & -0.00078 & 1.00977 & -0.00003 \\
\hline
\end{tabular}

energy-dependent analysis is required to explain the origin of the difference in $k_{\text {eff }}$. An example shown in Table 3 shows that the differences in the capture cross sections between JEFF-3.2 and ENDF-VII.1 cause significant differences in $k_{\text {eff }}$ below $200 \mathrm{keV}$ (equally distributed below and above $100 \mathrm{keV}$, the upper limit of the RRR in ENDF/B-VII.1 compared to $200 \mathrm{keV}$ in JEFF-3.2 and JENDL-4.0), while the differences in JEFF-3.2 and JENDL-4.0 can be observed also above $200 \mathrm{keV}$. The differences due to inelastic cross sections are statistically significant, however of minor importance for MYRRHA. The differences for swapping individual reaction cross sections do not add up to the total difference for the isotope. This can be attributed to the "interference" between the reactions through spectrum perturbations.

\section{Conclusions}

The sensitivity and uncertainty analysis of the MYRRHA MOX critical core design has been presented, identifying the major sensitivity coefficients, the uncertainty in $k_{\text {eff }}$ and the contribution of the most relevant quantities to the $k_{\text {eff }}$ uncertainty, using different codes and nuclear data libraries. Differences smaller than $4 \%$ have been obtained for the sensitivity coefficients. However, considerable differences have been observed between $k_{\text {eff }}$ uncertainties calculated using different covariance matrix evaluations.

A comparison of experimental data and evaluated nuclear data libraries for ${ }^{208} \mathrm{~Pb}$ and ${ }^{209} \mathrm{Bi}$ has been carried out. Existing experimental transmission data available in the EXFOR database has been identified and compared against the JEFF-3.2, ENDF/B-VII.1 and JENDL-4.0 evaluations. JENDL-4.0 has been found to be the most accurate evaluation for both isotopes in the MYRRHArelevant energy range.
Finally, ENDF/B-VII.1 and JENDL-4.0 nuclear data libraries have been compared using the MYRRHA MOX fueled critical core model. Different parts of data (energy regions and/or reactions) in reference JEFF-3.2 files have been substituted by corresponding data from the other libraries and effects on the system multiplication factor have been observed.

Authors would like to express their gratitude to the European Commission for the partial financial support of the CHANDA project (FP7-Fission-2013-605203), where this work is framed.

\section{References}

[1] J. Engelen et al., Int. J. Hydrogen Energy 40, 15137 (2015)

[2] A. Stankovskiy et al., Nucl. Data Sheets 118, 513 (2014)

[3] EC Community Research and Development Information Service - CHANDA project. http:// www . cordis . europa. eu/project/rcn/110083_ en.html

[4] M.B. Chadwick et al., Nucl. Data Sheets 118, 1 (2014)

[5] G. Van den Eynde et al., J. Nucl. Sci. Technol. 52, 1053 (2015)

[6] A. Stankovskiy et al., SCKCEN/4463803 (2014)

[7] B.T. Rearden et al., ORNL/TM-2005/39 (2016)

[8] D.B. Pelowitz, LA-CP-14-00745 (2014)

[9] I. Kodeli, Nucl. Sci. Eng. 138, 45 (2001)

[10] I. Kodeli et al., Proceedings of the $22^{\text {nd }}$ International Conference on Nuclear Energy for New Europe (2013)

[11] M.B. Chadwick et al., Nucl. Data Sheets 107, 2931 (2006)

[12] M.B. Chadwick et al., Nucl. Data Sheets 112, 2887 (2011)

[13] OECD-NEA Data Bank, USCD-1236/03 (2011)

[14] O. Iwamoto et al. J. Korean Phys. Soc. 59, 1224 (2011)

[15] M. Herman et al. BNL-94830-2011 (2011)

[16] OECD-NEA Data Bank JEFF-3.2 Evaluated Data Library - Neutron data. http://www.oecdnea.org/dbforms/data/eva/evatapes/jeff_32/

[17] K. Shibata et al., J. Nucl. Sci. Technol. 48, 1 (2011)

[18] N. Otuka et al., Nucl. Data Sheets 120, 272 (2014)

[19] N.M. Larson, ORNL/TM-9179/R8 (2008)

[20] R.E. MacFarlane et al., LA-12740-M (1999)

[21] L. Fiorito, OECD-NEA JEF/DOC-1726 (2015)

[22] R.F. Carlton, Bull. Am. Phys. Soc. 36, 1349 (1991)

[23] J.A. Harvey, EXFOR \# 13732.002 (1999)

[24] J.A. Harvey, EXFOR \# 10449.003 (1975) 